\title{
Two Distinct Regions in a Pulsed-Axisymmetric Jet
}

\author{
Hariyo P. S. Pratomo ${ }^{1}$, Klaus Bremhorst ${ }^{2}$ \\ ${ }^{1}$ Department of Mechanical Engineering \\ Petra Christian University \\ Siwalankerto Street 121 - 131 - Surabaya, 60236 East Java (Indonesia) \\ Fax number:+62 31 8417658, e-mail: hariyo_p@peter.petra.ac.id \\ 2 Department of Mechanical Engineering - the University of Queensland \\ Mansergh Shaw Engineering, St. Lucia, 4072 Queensland (Australia)
}

\begin{abstract}
This research concerns on the axial measurement of a pulsed-axisymmetric jet to investigate the decay of inverse of mean centerline axial velocity with the use of a single normal hot-wire anemometer. The measurement is performed by controlling mean exit velocity and pulsing frequency.

The axial measurement reveals that the pulsation method leads to the appearances of two distinct regions namely the pulsed dominated- and the high turbulence steady jetregions. However, the existence of the two regions can be controlled by adjusting the mean exit velocity and pulsing frequency. At a high mean exit velocity and low pulsing frequency, the high turbulence steady region is not seen, hence the transition region can not be found. Furthermore, at a high pulsing frequency and a constant mean exit velocity, the pulse dominated region becomes shorter.

The present study has provided a comprehensive knowledge in that the trend of the decay rate is very clear under the wide range of pulsation method imposed on the jet. This inclusive understanding becomes a substantial consideration for mixing processes as well as can be used to verify the $\mathrm{k}-\varepsilon$ turbulence model to predict the decay of normalized mean centerline-axial velocity.
\end{abstract}

Keywords. Pulsed-axisymmetric jet, pulsation method, decay rate of mean centerline velocity, hot-wire anemometer.

\section{Introduction}

In many widespread industrial applications such as mixing processes, unsteady jets can be of a considerable practical interest due to their more enhanced mixing and entrainment characteristics when compared to steady jets ([1], [2], [4], [5], [6], [10], [11], [12], [13], [20], [21], [23]). Such jets can be produced through mechanically (for example:, [4], [5], [6], [20]) or acoustically perturbation techniques (for example: [10], [18], [25]).

Of various types of mechanically perturbed jet, a fully pulsed jet which is generated by a pulsing valve is of the most important consideration in a number of studies since such a jet has higher level of entrainment than the other jets ([2], [20]). However, the jet still provides little structural information in terms of its axial properties due to limited measurements performed in the past ([3], [4], [6], [9], [12], [22], [23]).

An early investigation in the fully pulsed jet with a single mean exit velocity, $U_{e}$ of $36.6 \mathrm{~m} / \mathrm{s}$ and pulsing frequency, $\mathrm{f}_{\mathrm{p}}$ of $10 \mathrm{~Hz}$ ([4]) revealed a striking interesting phenomenon which is shown by the suddenly change of decay growth of inverse of axial-centerline velocity, $\mathrm{U}_{\mathrm{e}} / \mathrm{U}_{0}$ with a wider area of axial measurement (x/d up to 100 diameters downstream) were performed from a previous study ( $\mathrm{x} / \mathrm{d}$ up to 17 diameters downstream) with a constant mean exit velocity, $U_{e}$ of $36.6 \mathrm{~m} / \mathrm{s}$ and varying pulsing frequencies, $\mathrm{f}_{\mathrm{p}}$ of 10 and 25 $\mathrm{Hz}([6])$. At the upstream positions $(\mathrm{x} / \mathrm{d}<50)$ the decay rate is slower than that of a steady jet while after the downstream position, $\mathrm{x} / \mathrm{d}$ of 50 the decay growth switches to that of a steady jet.

A few years later, [23] continued the pulsed jet study with a constant mean exit velocity, $U_{e}$ of $35 \mathrm{~m} / \mathrm{s}$ and varying pulsing frequencies, $\mathrm{f}_{\mathrm{p}}$ of $5,10,15$, and 25 Hz. The researcher also found the similar interesting results with those of [4] associated with the suddenly change of decay rate but is of different scales with the results of [4] over 100 diameters downstream. Subsequently, [3] simulated the previous results [4] over 100 diameters downstream using the $\mathrm{k}-\varepsilon$ turbulence model. Nevertheless, they found that this model shows significant discrepancy in the further downstream regions but is consistent with the experimental results in the downstream regions ( $\mathrm{x} / \mathrm{d}$ below 50$)$, therefore, the change in slope of the centerline velocity decay of a fully pulsed jet still needs more explanation.

Continuing the former works ([3], [4], [23]), [12] experimentally investigated the fully pulsed jet with a single mean exit velocity, $\mathrm{U}_{\mathrm{e}}$ of $36.6 \mathrm{~m} / \mathrm{s}$ and pulsing frequency, $\mathrm{f}_{\mathrm{p}}$ of $10 \mathrm{~Hz}$ over some downstream distances $(40<\mathrm{x} / \mathrm{d}<80)$ which are considered to be the transition regions of decay growth. It was shown that the change in the decay rate is not clearly seen over this region.

Furthermore, [22] experimentally examined the fully pulsed jet up to 100 diameters downstream with a wider range of mean exit velocity $\left(10 \mathrm{~m} / \mathrm{s} \leq \mathrm{U}_{\mathrm{e}} \leq 35 \mathrm{~m} / \mathrm{s}\right)$ and a constant pulsing frequency, $f_{p}$ of $10 \mathrm{~Hz}$. [22] confirmed that the change in the decay rate was found.

In attempts to verify the experimental results of [22], [9] studied the pulsed jet with a range of mean exit velocity $\left(10 \mathrm{~m} / \mathrm{s} \leq \mathrm{U}_{\mathrm{e}} \leq 36.6 \mathrm{~m} / \mathrm{s}\right)$ and a constant pulsing frequency, $f_{p}$ of $10 \mathrm{~Hz}$ over 100 diameters downstream. However, the most recent results do not confirm the existence of transition region of the change in the decay rate of a fully pulsed jet.

Considering the abovementioned existing problems, it is the purpose of this present study to broaden the knowledge of a fully pulsed by carrying out an axial measurement up to further downstream in order to study the decay of inverse of mean centerline axial velocity. In line with the goal of this current work, the effects of varying mean exit velocity and pulsing frequency on the basic properties of the jet are 
investigated in a wider range than that of the previous works. This present work will provide the opportunity for more comprehensive understanding of pulsed jet than previously. For mixing process, the present existing results of the decay rate will benefit for evaluating the entrainment of the jet.

\section{Research Methodology}

The measurements were performed in the Pulsed Jet Laboratory at the Department of Mechanical Engineering, The University of Queensland. Natural or forced ventilation was not used during the measurements to avoid external effects which might be able to change the flow characteristics. The jet obtained air supply from the department main compressor which can provide the maximum compressed air at up to $600 \mathrm{kPa}$ (gauge).

Before passing a flow on/off valve, the compressed air is filtered by a coarse filter. After the valve, the compressed air is subsequently dried and filtered by the fine filter to avoid contaminants which can break the hot-wire sensor. A pressure reducing valve which is a pressure regulator to control the flow rate then feeds the air into the flow system which is connected by a $25 \mathrm{~mm}$ hose. An orifice plate designed by [8] to [7] was installed to measure the flow rate which corresponds to the desired mean exit velocities.

The fully pulsed jet was produced with apparatus as illustrated in Figure 1. The nozzle diameter is $12.77 \mathrm{~mm}$ with the pulsing valve driven by a pair of geared rollers of a 1:2 on-off ratio to generate an intermittent flow. A $200 \mathrm{~mm}$ disc was mounted on the nozzle to avoid directly entrainment of the fluid behind the nozzle, hence allowing the flow near the jet exit to be axisymmetric. To obtain the required pulsing frequency, the shaft of one roller was coupled to a variable speed DC motor, the speed of which can be adjusted.

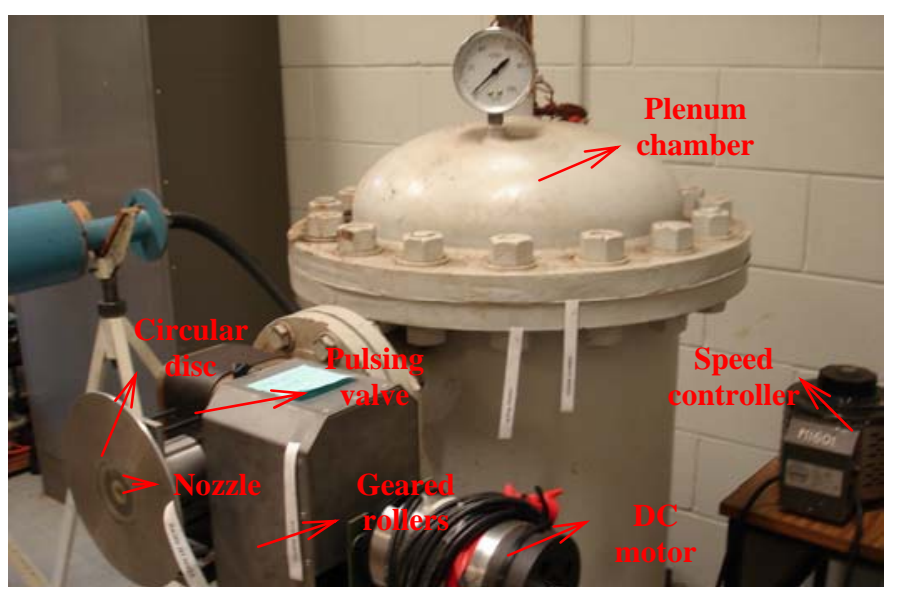

Figure 1. Pulsed jet apparatus

A plenum chamber was used to stabilize flow variations caused by the opening and closing of the pulsing valve. The chamber can withstand pressure up to $100 \mathrm{kPa}$ (gauge). For this current research, the pressure was measured with a pressure gauge and was found to be in a range of $2.5-30 \mathrm{kPa}$ (gauge) which corresponds to the desired exit velocities. The pressure fluctuations in the chamber, as a result of the pulsing flows, never exceeded $2 \%$ of the chamber gauge pressure ([14]), therefore their effects on the exit flow can be neglected. From observations during the experiments, the level of the pressure fluctuations decreased with increasing pulsing frequency.

Turbulent flow generated by the jet was studied with the use of a single-normal hot-wire anemometer operated in a constant-temperature mode. The axial measurement was performed up to 100 diameters downstream. A larger range of mean exit velocities, $U_{e}$ of 13.7, 20.6, 27.6, 34.4, and $41.2 \mathrm{~m} / \mathrm{s}$ and pulsing frequencies, $\mathrm{f}_{\mathrm{p}}$ of $5,10,15$, and $25 \mathrm{~Hz}$ were used to have comparable results with those of previous experimenters ([4], [6], [9], [12], [22], [23]).

The hot-wire anemometer system incorporates a single normal hot-wire probe, DISA 55M01 main unit, 55M11 CTA booster adapter, and 55M05 power pack. The probe was a single normal wire made of Sigmond Cohn alloy $851(79 \% \mathrm{Pt}, 15 \% \mathrm{Rh}$, and $6 \% \mathrm{Ru})$ mounted on the prongs with a spot-welding technique performed at the Mechanical Instrument Laboratory, the Department of Mechanical Engineering, the University of Queensland having the ultimate strength, temperature coefficient of resistivity, $\alpha_{20}$, resistivity, $\chi_{20}$, of $1.724 \times 10^{6} \mathrm{kPa}, 0.7 \times 10^{-3}$ ${ }^{\circ} \mathrm{C}^{-1}$, and $30 \times 10^{6} \Omega-\mathrm{cm}$, respectively. The wire size was 2 $\mathrm{mm}$ long and $10.16 \mu \mathrm{m}$ diameter. During the measurements, this probe was placed with the probe stem parallel to the mean flow direction to measure the axial velocity components.

Signal processing and data logging devices for digital sampling technique include a Tektronix 2201 oscilloscope and $8050 \mathrm{~A}$ digital voltmeter, two $737 \mathrm{M}$ low-pass (L/P) filters and amplifiers, two signal conditioning $\quad(715 \mathrm{~A} / \mathrm{B}$ and $715 \quad \mathrm{~A} / \mathrm{D}$ inverter/amplifier/summer) units, an analog to-digital (A/D) card, and a personal computer equipped with a data logging software. For optimum sampling criteria, the hot-wire signal was sampled with the sampling rate of $1000 \mathrm{~Hz}$ and the number of sample of 10000 .

Before the execution of the primary experiments, several experimental procedures were to be achieved, including hot-wire calibration, optimum digital sampling technique, pulsed jet symmetry check. The hotwire calibration has been performed within a high degree of accuracy, giving a $\pm 0.03 \%$ accuracy for the extended power-law equation improved by the look-up table method and $1.22 \% \mathrm{rms}$ relative error of the fluctuating velocity component, u'. Based on the optimum digital sampling evaluation, the appropriate sampling parameters are a $1000 \mathrm{~Hz}$ sampling frequency; 50,000 number of samples, and 50 seconds sampling time, allowing a $0.26 \%$ uncertainty of the estimated mean value, $\varepsilon[U]$. Furthermore, the jet centerline determination shows that the pulsed jet follows a straight line trajectory when propagating downstream. Also, a high degree of the radial profile symmetry in the horizontal and vertical direction is proved by the radial profile test of the jet.

\section{A. Basic Equations}

From the Reynolds decomposition, the velocity components of the pulsed jet can be mathematically expressed as 


$$
U_{i}=U+u
$$

where $U_{i}$ is the instantaneous axial velocity, $U$ is the mean axial velocity, and $u$ is the aggregate axial turbulence. The flow is statistically stationary for the cases of spatial averaging which are demonstrated by the results of $\bar{u} \equiv 0$. The mean axial velocity in (1) can be determined from a discrete equation applicable to digital signal sampling over a finite time period as in

$$
\hat{U}=\frac{\sum_{i=1}^{n} U_{i}}{N}
$$

where $\mathrm{U}_{\mathrm{i}}$ is the $\mathrm{i}^{\text {th }}$ sampled signal, $\mathrm{N}$ is the total number sampled. $\hat{U}$ is an estimation of $U$ and it holds that $\hat{U}=U$ as a consequence of the statistically stationary flow.

The equation of the decay rate of mean centerline velocity formulated by [4] is expressed as in

$$
\frac{U_{0}}{U_{e}}=\frac{A_{1}}{\left(\frac{x}{d}+\frac{x_{01}}{d}\right)}
$$

where $U_{e}$ is the mean exit velocity, $U_{0}$ is the mean centerline velocity, $A_{1}$ is a decay constant, $x_{01} / d$ is the non-dimensional effective origin for $\mathrm{U}_{0}, \mathrm{x}$ is the axial coordinate and $\mathrm{d}$ is the nozzle diameter.

To evaluate the normalized jet volume flow rate formulated over $0<x / d<20$, [6] and [14] introduced an empirical equation as in

$$
\frac{Q}{Q_{e}}=\frac{A_{1} A_{2}^{2}}{\alpha} \frac{\left(x+x_{02}\right)^{2}}{d\left(x+x_{01}\right)}
$$

where $Q_{e}$ is the mean jet volume calculated from a flow meter, A2 is the spreading rate constant, $x_{02}$ is the dimensional effective origin which may not essentially be the same as $\mathrm{x}_{01}$, and $\alpha$ is a constant. Equation (4) is valid both for the steady and fully pulsed jet cases by consideration that both of the jets grow linearly to the downstream regions

\section{Results and Discussion}

Figure 2 demonstrates one of the complete results of the decay of mean centerline axial velocity, $\mathrm{U}_{0}$ normalized by the mean exit velocity, $\mathrm{U}_{\mathrm{e}}$ along 100 diameters downstream. The mean exit velocity was $34.4 \mathrm{~m} / \mathrm{s}$ and the pulsing frequency was increased from 5 to $25 \mathrm{~Hz}$. It is clearly seen that the changes in the decay rate are found in the pulsed jet displaying two distinct regions namely the pulse dominated- and the high turbulence steady jetregions (at the pulsing frequencies, $f_{p}$ of 10,15 , and 25 $\mathrm{Hz}$ ). However, the change in the decay rate can not be identified at the lowest pulsing frequency of $5 \mathrm{~Hz}$. From the figure, the pattern is very clear that the location of the change in the decay rate shifts upstream for the higher

\footnotetext{
${ }^{1}$ For the original symbols of this equation, the reader can see Bremhorst and Harch (1979) page 45. The formulation of this equation is amended to facilitate consistent expressions with the symbols used throughout this paper.
}

pulsing frequencies as a result of the increasing pulsing frequency with the mean exit velocity was kept constant.

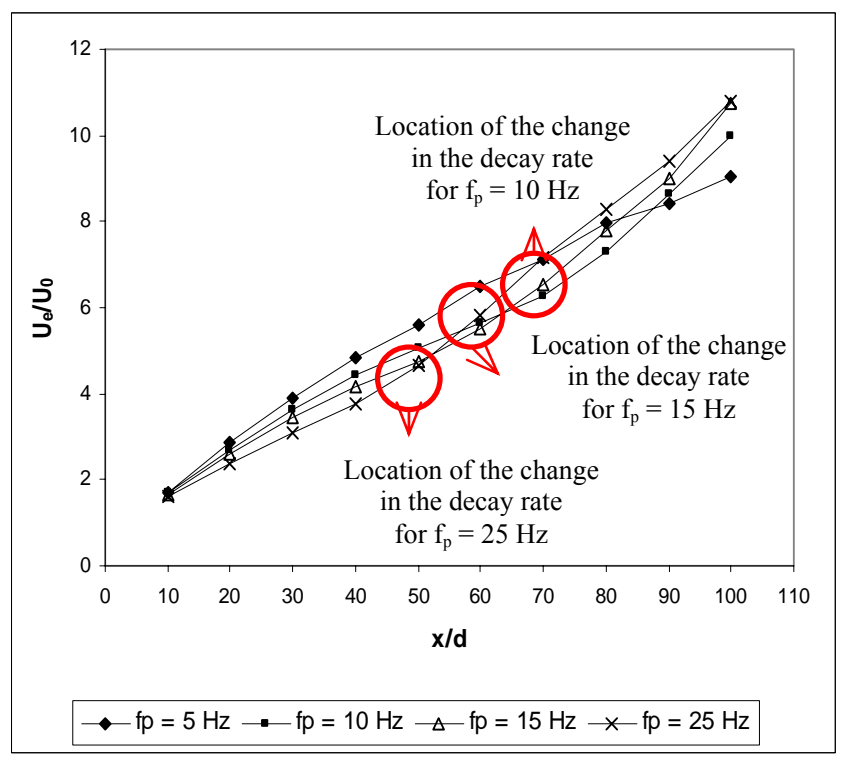

Figure 2. Decay of normalized mean centerline axial velocity at $\mathrm{U}_{\mathrm{e}}=34.4 \mathrm{~m} / \mathrm{s}$

The existence of the aforementioned distinct regions is also proved as in Figure 3 which is one of the complete results of which the mean exit velocity is varied under a constant pulsing frequency. The two distinct regions are clearly seen at the lowest mean exit velocity of $13.7 \mathrm{~m} / \mathrm{s}$. It is clearly seen that as the mean exit velocity is augmented under a constant pulsing frequency, the high turbulence steady jet region can not be identified.

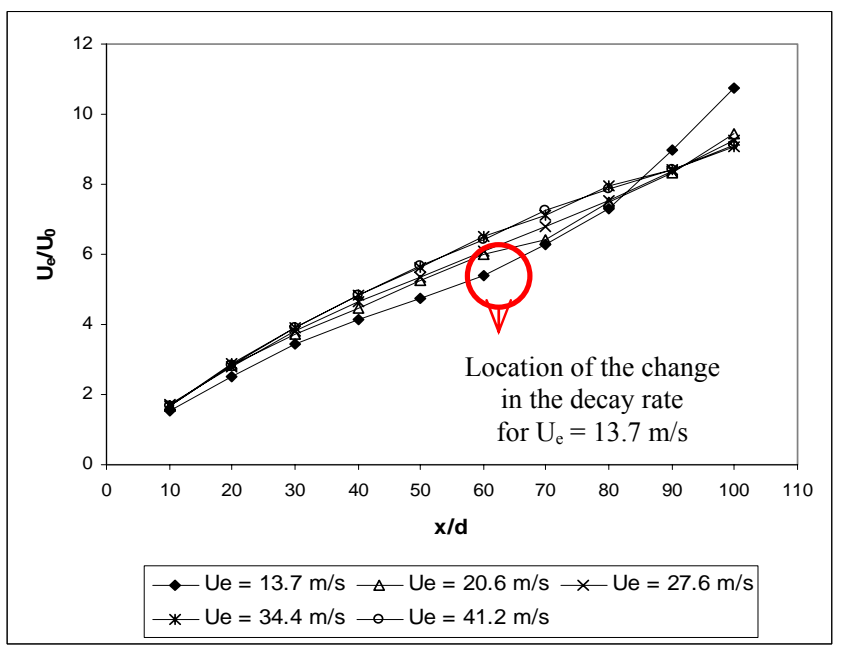

Figure 3. Decay of normalized mean centerline axial velocity at $\mathrm{f}_{\mathrm{p}}=5 \mathrm{~Hz}$

Figure 4 illustrates comparisons of the decay of mean centerline axial velocity, $\mathrm{U}_{0}$ normalized by $\mathrm{U}_{\mathrm{e}}$ between the existing results of the pulsed and steady jets. From the figure, it is seen that the patterns of the decay among the researchers ([4], [23], the present researcher) are the same indicating that there is a significant change in the decay of mean centerline velocity in the pulsed jet, thus there are two distinct regions. [6] did not find the significant change in the decay as they only performed 
their measurement in the first 17 diameters downstream. Yet, the present results are a continuation of [6]'s results in the near field region. Considering the results of [12], the significant change in the decay rate is less evident as [12]'s investigation only covered a limited axial measurement $(40<\mathrm{x} / \mathrm{d}<80)$. However, the evidence of significant change in the decay of [12]'s result can be still be seen from the two different line slopes which coincide with each other at $\mathrm{x} / \mathrm{d}$ around 60 .

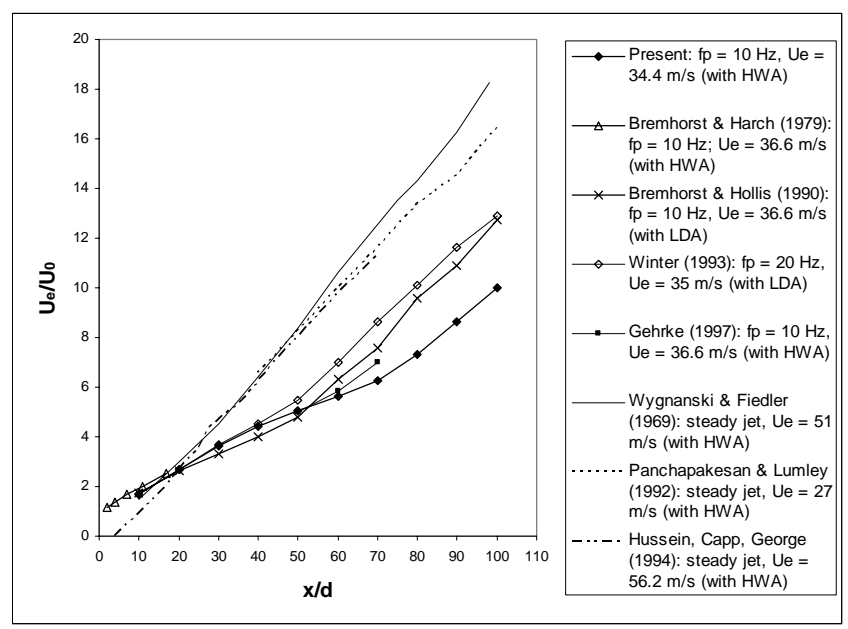

Figure 4. Comparison of the decay of normalized mean centerline axial velocity between the pulsed and steady jets ${ }^{2}$

Furthermore, the differences in the levels of $\mathrm{U}_{\mathrm{e}} / \mathrm{U}_{0}$ between the results of [4] and the present researcher may be due to a different sensor used. The present results are obtained with a hot-wire sensor while [4] used a laser Doppler anemometer. The differences in the levels of $\mathrm{U}_{\mathrm{e}} / \mathrm{U}_{0}$ are also seen for the present results and [23]'s results. Instead of the different sensor used, this is because of the different pulsing frequency imposed on the jet. Also, as shown in Figure 4, the decays in the near field region $(\mathrm{x} / \mathrm{d}<50)$ are relatively the same for various researchers ([6], [12], [23], the present researcher). Moreover, the gradients of the decay in the far downstream region $(\mathrm{x} / \mathrm{d}>50)$ are parallel to each other ([23], [12], the present researcher).

Viewing the gradients of the decay of steady jets ([17], [19], [24]), the present researcher, [4], [12], and [23] show the same gradients to those of the decay rate of steady jets. Therefore, the pulsed jets do turn into steady jets after the region of $\mathrm{x} / \mathrm{d}$ beyond 50 .

Figure 5 illustrates comparisons of the decay of the present study with the previous works by [6], [23], and the existing results of steady jets ([17], [19], [24]). Again, the similar patterns of the decay of mean centerline axial velocity in the pulsed jet as illustrated in Figure 5 appear among the pulsed jet researchers ([23], the present researcher). Viewing the result of [6], the present result is a continuation of the previous result in the near field region $(\mathrm{x} / \mathrm{d}<50)$. Comparing the present results with [23]'s work, the differences in the levels of $\mathrm{U}_{\mathrm{e}} / \mathrm{U}_{0}$ are because of the different sensor and pulsing frequency used. Considering the three previous researchers ([17], [19], [24]) of steady jets, the slopes of

${ }^{2}$ HWA: Hot-wire anemometer; LDA: laser Doppler anemometer the decay rate of the present researcher and [23] in the far downstream region $(\mathrm{x} / \mathrm{d}>50)$ are seen relatively the same.

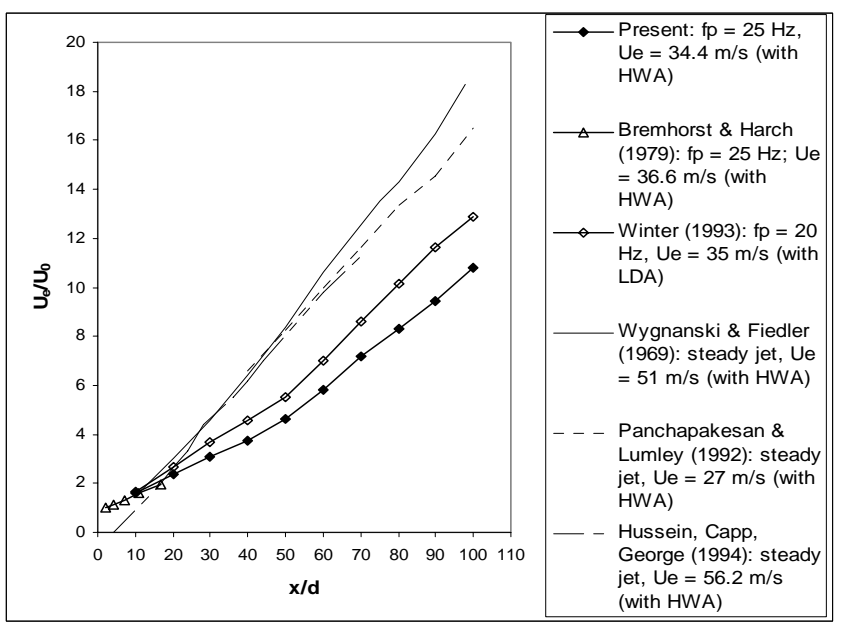

Figure 5. Comparison of the decay of normalized mean centerline axial velocity between the pulsed and steady jets ${ }^{1}$

Moreover, as shown in the figures, the transition regions of the present results are found not as sharp as the results of the early findings of [4], [16], and [23] showing no identifiable 'kink'. This indicates that the pulsed jet does not undergo a sudden change in the decay rate but the change grows gradually. [12] also demonstrates such an evidence in his transition region $(40<\mathrm{x} / \mathrm{d}<80)$.

Table 1 summarizes the constants and effective origin values of the decay of normalized mean axial velocity over 100 diameters downstream. The values are obtained from equation (3) (introduced by [4] and [6]) by linear curve fitting (for example, illustrated in Figures 6) as the decay of the pulsed jet in both of the two distinct regions is relatively linear. The linear decay rates of the present work confirm the existing empirical evidences ([9], [12], [13], [14], [16], [22], [23]) as their pulsed jets have a linear decay rate over the pulsed dominated region $(10 \leq \mathrm{x} / \mathrm{d} \leq 50)$. The similar trend of the decay rate also holds in the locations beyond the pulsed dominatedregion $(\mathrm{x} / \mathrm{d}>50)$ as found by the present and earlier works ([4], [23]) as in Figure 4 and 5.

As can be seen from the table, the values of the jet effective origin, $x_{01} / d$ of the pulsed jet in the near field region are always positive. This occurs since the unsteady effects generated by the pulsing frequency shift upstream the effective origin of the pulsed jet. On the contrary, the $\mathrm{x}_{01} / \mathrm{d}$ values in the far downstream region always poses negative values as demonstrated by the steady jets ([12], [13], [15], [16], [23], [24]). This indicates that the pulsed jet has switched into a steady jet as a result of the lessening unsteady effects. Moreover, the values of $A_{1}$ and $x_{01} / d$ vary accordingly as the pulsing frequency and mean exit velocity change. In terms of the calculation of the jet entrainment, equation (4) can not be used as a consequence of the existence of the two distinct regions giving two values of $\mathrm{A}_{1}$ and $\mathrm{x}_{01} / \mathrm{d}$ under the corresponding controlled parameters (see the table). However, whenever only does the pulsed dominatedregion appear, equation (4) holds to evaluate the jet entrainment in the near region of the jet. 
Table 1. Decay constants of inverse of mean centerline axial velocity of the pulsed jet

\begin{tabular}{|c|c|c|c|c|}
\hline $\begin{array}{c}\text { Exit } \\
\text { Velocity, } \\
\mathrm{U}_{\mathrm{e}}(\mathrm{m} / \mathrm{s}) \\
\end{array}$ & $\begin{array}{c}\text { Pulsing } \\
\text { Frequency, } \\
\mathbf{f}_{\mathrm{p}}(\mathrm{Hz}) \\
\end{array}$ & $\begin{array}{c}\text { Range of } \\
x / d\end{array}$ & $\mathbf{A}_{1}$ & $x_{01} / d$ \\
\hline \multirow{8}{*}{13.7} & \multirow[t]{2}{*}{5} & $10-70$ & 13.11 & 12.56 \\
\hline & & $80-100$ & 5.81 & -37.64 \\
\hline & \multirow[t]{2}{*}{10} & $10-50$ & 13.03 & 9.12 \\
\hline & & $60-100$ & 8.1 & -13.68 \\
\hline & \multirow[t]{2}{*}{15} & $10-40$ & 13 & 7.52 \\
\hline & & $50-100$ & 7.97 & -10.55 \\
\hline & \multirow[t]{2}{*}{25} & $10-30$ & 14.19 & 7.80 \\
\hline & & $40-100$ & 7.77 & -7 \\
\hline \multirow{8}{*}{20.6} & \multirow[t]{2}{*}{5} & $10-70$ & 12.65 & 14.86 \\
\hline & & $80-100$ & 10.21 & -4.07 \\
\hline & \multirow[t]{2}{*}{10} & $10-50$ & 13.27 & 12.15 \\
\hline & & $60-100$ & 8.13 & -14.45 \\
\hline & \multirow[t]{2}{*}{15} & $10-40$ & 14.16 & 12.55 \\
\hline & & $50-100$ & 7.74 & -15.30 \\
\hline & \multirow[t]{2}{*}{25} & $10-30$ & 14.52 & 11.46 \\
\hline & & $40-100$ & 7.81 & -10.79 \\
\hline \multirow{7}{*}{27.6} & 5 & $10-100$ & 12.39 & 14.77 \\
\hline & \multirow[t]{2}{*}{10} & $10-60$ & 12.97 & 13.56 \\
\hline & & $70-100$ & 8.03 & -18.68 \\
\hline & \multirow[t]{2}{*}{15} & $10-60$ & 13.11 & 12.34 \\
\hline & & $70-100$ & 8.2 & -13.71 \\
\hline & \multirow[t]{2}{*}{25} & $10-40$ & 13.99 & 11.93 \\
\hline & & $50-100$ & 8.21 & -9.32 \\
\hline \multirow{7}{*}{34.4} & 5 & $10-100$ & 12.42 & 16.95 \\
\hline & \multirow[t]{2}{*}{10} & $10-60$ & 13.32 & 15.93 \\
\hline & & $70-100$ & 7.4 & -25.97 \\
\hline & \multirow[t]{2}{*}{15} & $10-50$ & 13.32 & 14.04 \\
\hline & & $60-100$ & 7.21 & -23.66 \\
\hline & \multirow[t]{2}{*}{25} & $10-40$ & 13.94 & 12.79 \\
\hline & & $50-100$ & 8.22 & -11.82 \\
\hline \multirow{7}{*}{41.2} & 5 & $10-100$ & 12.32 & 16.39 \\
\hline & \multirow[t]{2}{*}{10} & $10-70$ & 12.88 & 20.02 \\
\hline & & $80-100$ & 8.07 & -22.39 \\
\hline & \multirow[t]{2}{*}{15} & $10-60$ & 13.17 & 13.86 \\
\hline & & $70-100$ & 7.56 & -22.16 \\
\hline & \multirow[t]{2}{*}{25} & $10-40$ & 13.47 & 12.69 \\
\hline & & $50-100$ & 7.85 & -14.57 \\
\hline
\end{tabular}

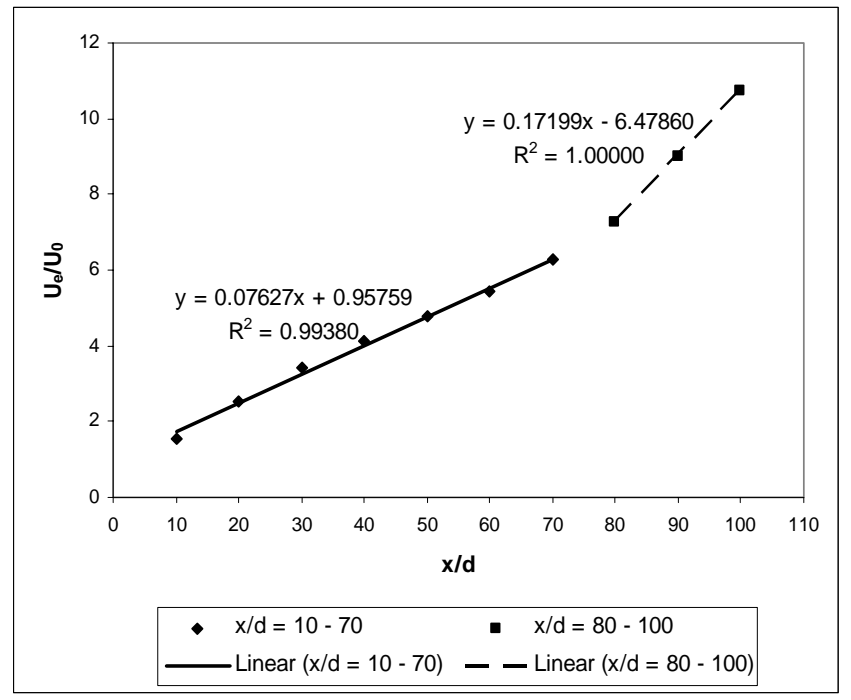

Figure 6. Linear curve-fit of the decay of normalized mean centerline velocity at $U_{e}=13.7 \mathrm{~m} / \mathrm{s}$ and $f_{p}=5 \mathrm{~Hz}$
In terms of the slopes of the decay in the pulsed dominated region, the present results confirm the findings by [2] and [6] explaining that the decay rate of the mean centerline velocity is strongly dependent on the pulsing frequency and level of excitation. From the present results, it is demonstrated that the slower decay rates appear at the higher pulsing frequencies and lower mean exit velocities than those at the lower pulsing frequencies and higher mean exit velocities. Consequently, the effective origins (associated with the constants of $x_{01} / d$ ) at the higher pulsing frequencies and lower mean exit velocities would be moved more upstream (having a smaller value of $\mathrm{x}_{01} / \mathrm{d}$ ) than those of the lower pulsing frequencies and higher mean exit velocities in which these are associated with the slightly steeper slope of the decay rate for the lower pulsing frequencies and higher mean exit velocities.

In addition, the present results also agree with the findings of [5] demonstrating that unsteady jets have slower decay rates than those of steady jets. This is caused by interactions between the jet fluid and the pressure field during each cycle of pulsation leads to negative pressure gradients permitting increasing momentum and large fluctuation of the periodic components ([2], [6], [25] $\left.]^{3}\right)$ under a high pulsing frequency. [16] and [23] also demonstrate a significantly increasing jet momentum in the near field of the jet exit.

\section{Conclusion}

The experimental work has been to extend the range of the parameters previously considered, thereby having filled a gap in the pulsed jet research in that a larger range of mass flow rates and pulsing frequencies have been used than previously.

The pulsation method imposed on the pulsed jet significantly affects the whole jet flow field. This is associated with the existence of the two distinct regions namely the pulsed dominated and high turbulence steady jet region depending on the level of mean exit velocity and pulsing frequency. After the attenuation of periodic pseudo velocity component indicating that the pulsed dominated region has ended, the pulsed jet behaves like a steady jet but with much higher turbulence intensity levels after the transition region. However, the transition region would not be seen at a high mean exit velocity and a low pulsing frequency. Moreover, the pulsed jet would start to switch to a steady jet earlier at a high pulsing frequency due to the small amplitude of centerline axial fluctuating components; hence, the pulse dominated region become shorter.

In terms of the scope of this project, this study does not cover the $\mathrm{k}-\varepsilon$ turbulence modelling to predict the decay rate of the jet. However, the existing inclusive results of the decay rate are worthwhile to verify the $\mathrm{k}-\varepsilon$ model as developed by [3] in terms of its failure to predict the decay rate in the pulsed jet. Regarding the uniqueness of the jet structure under the controlled parameters imposed, this however deters the entrainment evaluation using the equation suggested by [6] as there

\footnotetext{
${ }^{3}$ This work is a continuation of the same work in 1980 which focused on the investigation of coherent structure dynamics of vortex pairing.
} 
are two decay constants, $\mathrm{A}_{1}$ and two non-dimensional effective origins, $x_{01} / d$ as a result of the two distinct regions. Even so, when the pulsed dominated-region entirely appears in the jet structure the empiric equation by [6] can be used to calculate the jet entrainment.

The existing results of the decay rate, hence, are of considerable practical interest for mixing process as the patterns are very obvious. Varying the mass flow rate and pulsing frequency of the jet in proper use can be implied to control the effectiveness of mixing process as the slope of the decay rate under the corresponding controlled parameters affects the decay constant as well as the non-dimensional effective origin in the pulsed dominated region.

\section{Acknowledgement}

This research project was supported by grants from the Technological and Professional Skills Development Sector Project ADB Loan No. 1792 INO.

The first author is indebted and grateful to Prof. Klaus Bremhorst, AM for introducing him to the field of hot-wire anemometry and for every inspiring and valuable discussion during this research and to $\mathrm{Mr}$. George Dick who rendered assistance in repairing hotwire sensor.

\section{References}

[1] Binder, G., M. Favre-Marinet, A. Craya, Hac Te Veug (1973). Jet Instationnaires, Labor de Mecanique des Fluides, Universite de Grenoble

[2] Bremhorst, K. (1979). "Unsteady Subsonic Turbulent Jets." Recent Developments in Theoretical and Experimental Fluid Mechanics, Springer Verlag, Berlin: 480-500.

[3] Bremhorst, K. and L. J. W. Graham (1993). "Application of the k-e Turbulence Model to the Simulation of a Fully Pulsed Free Air Jet." Transactions of the ASME, Journal of Fluids Engineering 115: 70-74.

[4] Bremhorst, K. and P. G. Hollis (1990). "Velocity Field of an Axisymmetric Pulsed, Subsonic Air Jet." AIAA Journal 28, no. 12: 2043-2049.

[5] Bremhorst, K. and R. D. Watson (1981). "Velocity Field and Entrainment of a Pulsed Core Jet." Journal of Fluids Engineering 103, no. 4: 605-608.

[6] Bremhorst, K. and W. H. Harch (1979). "Near Field Velocity Measurements in a Fully Pulsed Subsonic Air Jet." Turbulent Shear Flows I, Springer Verlag, Berlin: 3754.

[7] BS1042 (1964). Orifice Plates, Nozzles and Venturi Tubes. Methods for the Measurement of Fluid Flow in Pipes, British Standards Institute. London.

[8] Bullock, S. B. (1981). Annular Air Jet Investigation. UG Thesis, Department of Mechanical Engineering. Brisbane, The University of Queensland, Australia.

[9] Cocchis, P. (2004). Experimental Investigation of the Velocity Field of a Fully Pulsed Jet. Master Thesis, Department of Mechanical Engineering. Lyngby, The Technical University of Denmark, Denmark: 234 pages.

[10] Crow, S. C. and F. H. Champagne (1971). "Orderly Structure in Jet Turbulence." Journal of Fluid Mechanics 48, part 3: $547-591$.

[11] Curtet, R. M. and J. P. Girard (1973). Visualisation of a Pulsating Jet. Proceedings of the ASME Symposium on the Fluid Mechanics of Mixing, Atlanta, the United States of America.
[12] Gehrke, P. J. (1997). The Turbulent Kinetic Energy Balance of a Fully Pulsed Axisymmetric Jet. PhD Thesis, Department of Mechanical Engineering. Brisbane, The University of Queensland, Australia: 378 pages.

[13] Graham, L. J. W. (1991). Development of Experimental and Computational Techniques for Investigation of a Heated Pulsed Air Jet. PhD Thesis, Department of Mechanical Engineering. Brisbane, The University of Queensland, Australia: 257 pages.

[14] Harch, W. H. (1977). Noise and Flow Measurements in a Pulsed Jet. PhD Thesis, Department of Mechanical Engineering. Brisbane, The University of Queensland, Australia.

[15] Hinze, J. O. (1975). Turbulence, McGraw-Hill, New York.

[16] Hollis, P. G. (1988). Velocity Field Investigation of A Fully Pulsed Air Jet with A Laser Doppler Anemometer. $\mathrm{PhD}$ Thesis, Department of Mechanical Engineering. Brisbane, The University of Queensland, Australia: 371 pages.

[17] Hussein, J. H., S. P. Capp, William K. George (1994). "Velocity Measurements in a High-Reynolds-NumberMomentum-Conserving, Axisymmetric, Turbulent Jet." Journal of Fluid Mechanics 258: 31- 75.

[18] Lovett, J. A. and S. R. Turns (1990). "Experiments on Axisymmetric Pulsed Turbulent Jet Flames." Journal 28, no. 1: 38-46.

[19] Panchapakesan, N. R. and J. L. Lumley (1993). "Turbulence Measurements in Axisymmetric Jets of Air and Helium, Part I: Air Jet." Journal of Fluid Mechanics 246: 197-223.

[20] Platzer, M. F., J. M. Simmons, K. Bremhorst (1978). "Entrainment Characteristics of Unsteady Subsonic Jets." AIAA Journal 16, no. 3: 282-284.

[21] Tanaka, Y. (1984). "On the Structure of Pulse Jet." Bulletin of JSME 27, no. 230: 1667-1674.

[22] Toh, C. S. (2001). Mean Velocity and Turbulence Measurements in a Pulsed Jet for a Range of Mass Flows. UG Thesis, Department of Mechanical Engineering. Brisbane, The University of Queensland, Australia: 155 pages.

[23] Winter, A. R. (1991). A Laser Doppler Anemometer Investigation into Fully Pulsed Jet Flows with an Examination of Velocity Bias Error. PhD Thesis, Department of Mechanical Engineering. Brisbane, The University of Queensland, Australia.

[24] Wygnanski, I. and H. Fiedler (1969). "Some Measurements in the Self-Preserving Jet." Journal of Fluid Mechanics 38: 577-612.

[25] Zaman, K. B. M. Q. and A. K. M. F. Hussain (1980). "Vortex Pairing in a Circular Jet under Controlled Excitation, Part 2: Coherent Structure Dynamics." Journal of Fluid Mechanics 101, part 3: 493-544. 